\title{
The Importance of Topology and Reconnection in Active Region CMEs
}

\author{
Robert J. Leamon $\dagger$ \\ L-3 Communications at NASA Goddard Space Flight Center, \\ Code 612.5, Greenbelt, MD 20771, USA \\ email: leamon@grace.nascom.nasa.gov
}

\begin{abstract}
A distinctive characteristic of interplanetary magnetic clouds is their rope-like magnetic structure, i.e., their smoothly-varying helical field lines whose pitch increases from their core to their boundary. Because this regular structure helps to make MCs particularly geoeffective, it is important to understand how it arises.

We discuss recent work which relates the magnetic and topological parameters of MCs to associated solar active regions. This work strongly supports the notion that MCs associated with active region eruptions are formed by magnetic reconnection between these regions and their larger-scale surroundings, rather than simple eruption or entrainment of pre-existing structures in the corona or chromosphere. We discuss our findings in the context of other recent works on both the solar and interplanetary sides, including ion composition and various MHD models of magnetic cloud formation.
\end{abstract}

Keywords. Sun: coronal mass ejections (CMEs) — Sun: solar-terrestrial relations

\section{Introduction}

Coronal mass ejections (CMEs) are an almost daily occurrence on the sun. Interplanetary manifestations of CMEs often have a very distinct magnetic structure, namely a large-scale helix. Burlaga et al. (1981) described this magnetic structure as a "Magnetic Cloud." According to Burlaga's definition, three properties are required to identify a structure as a magnetic cloud: $(i)$ a very low proton temperature; $(i i)$ a large, smooth, monotonic rotation of the field direction; and ( $i i i)$ enhanced magnetic field strength. The general case of a smooth, monotonic rotation of the field direction is called a magnetic flux rope. At least one-third (Gosling 1990), and, according to some researchers (e.g., Webb 2000) considerably more, of the interplanetary manifestations of CMEs observed in situ have magnetic flux rope (large-scale helix) structure.

When one can associate a solar source with interplanetary ejecta, the source is almost always an active region or a quiescent solar filament. At first inspection, those magnetic clouds spawned from ARs and filaments seem to be similar, but as we shall demonstrate, source-related differences exist.

\subsection{Magnetic Clouds Associated With Filaments}

The relationship between magnetic clouds and their solar progenitors may be much more straightforward for filaments (Bothmer \& Rust 1997; Crooker 2000) than active regions (Pevtsov \& Canfield 2001; Leamon et al. 2002), as we shall now discuss.

Left-handed (Right-handed) MCs associated with erupting filaments tend to come from the Northern (Southern) hemisphere - only about 1 in 30 filament eruptions violate this

$\dagger$ Work performed while at Department of Physics, Montana State University, Bozeman, MT 59717, USA. 
rule (Bothmer \& Rust 1997). The tilts of their axes with respect to the ecliptic plane are correlated with tilt angles (relative to the heliomagnetic equator) of the associated filaments. The north-south component of the magnetic fields inside the MC tends to reverse at or around cycle maximum; the field of MCs is made up of the large-scale dipole fields. In short, we can say that such MCs carry the imprint of the large-scale solar magnetic field out into the heliosphere.

\subsection{Magnetic Clouds Associated With Active Regions}

The same hemispheric preference for handedness holds for AR-associated CMEs, but only at about a 2:1 ratio, which is about the same as the handedness of active regions themselves (Joy's Law). However, the tilts of MC axes with respect to the ecliptic plane are not correlated with tilt angles (relative to the heliomagnetic equator) of associated ARs. The major difference between the two north-south component of MC fields tends to flip at around cycle minimum, with the polarity of sunspots. So unlike the global nature of filament-based eruptions, the field of active region MCs is made up both active-region and large-scale dipole fields, and such MCs carry the imprint of both active regions and the large-scale solar magnetic field out into the heliosphere.

\subsection{Outline}

In the next section we shall see that, for AR-associated eruptions at least, magnetic clouds are formed by magnetic reconnection between these regions and their larger-scale surroundings, rather than simple eruption of pre-existing structures in the corona or chromosphere. We shall then investigate what the plasma composition data can tell us about the nature and dynamics of that reconnection, and that composition highlights further differences between active region-based events and those associated with filaments.

\section{Magnetic Structure: Solar-Interplanetary Comparisons}

Following Lepping et al. (1990), we model magnetic clouds using the constant- $\alpha$ forcefree field solution of Lundquist (1950), which assumes cylindrical symmetry. In a cloudcentered frame of reference, the axial and tangential fields are modeled as zeroth- and first-order Bessel functions, respectively, and there is no radial component. Full details of our magnetic cloud model and code are described in Leamon et al. (2004), along with sample fits. Once a satisfactory fit is found, it is a simple matter to deduce current density from the induction equation, and calculate the integrated current and flux in the cloud. There are some (sometimes gross) approximations to be made in modeling interplanetary ejecta as an infinite right cylinder, as well as constant- $\alpha$; however, since we are comparing the helicity of magnetic clouds to that of solar active regions, which are represented by constant- $\alpha$ force-free field models (Pevtsov et al. 1995) the model and assumptions used are a valid first step.

Table 1, reproduced from Leamon et al. (2004), contains a comparison of flux, current and twist between 12 magnetic clouds and their identified progenitor active regions. We draw the reader's attention to four significant results: $(i)$ the flux ratios $\Phi_{M C} / \Phi_{A R}$ tend to be large; $(i i)$ the current ratios $I_{M C} / I_{A R}$ tend to be orders of magnitude less than the flux ratios $\Phi_{M C} / \Phi_{A R}$; ( $\left.i i i\right)$ there is a statistically significant proportionality between the flux and current ratios; and ( $i v$ ) in four of the 12 events (Nos. 3, 4, 11, and 12) the cloud and active region have opposing senses of twist.

These features can be discussed in the framework of three highly simplified, but conceptually useful, models of the solar genesis of magnetic clouds. These models are that the flux in the magnetic cloud originates in: (1) the active region alone (the AR model); (2) 
Table 1. Comparison of flux, current, and twist values in ARs and MCs.

\begin{tabular}{|c|c|c|c|c|c|c|c|c|}
\hline No. & Year & $\begin{array}{r}\text { Even } \\
\text { Eruption }\end{array}$ & $\begin{array}{l}\text { Times: } \\
\text { Magnetogram }\end{array}$ & $\mathrm{AR}$ & $\begin{array}{r}I_{M C} / I_{A R} \\
\times 10^{-3}\end{array}$ & $\begin{array}{r}\Phi_{M C} / \Phi_{A R} \\
\%\end{array}$ & $(\alpha L)_{A R}$ & $(\alpha L)_{M C}$ \\
\hline 1 & 1995 & Feb & b 04 01:51 & 7834 & $0.15-0.53$ & $20-38$ & -0.34 & 53.2 \\
\hline 2 & - & Feb 28 08:46 & Feb $2707: 17$ & 846 & 30 & $8-18$ & 01 & 62.2 \\
\hline- & - & - & Feb 28 19:45 & - & $0.12-0.28$ & $8-12$ & -0.91 & \\
\hline 3 & - & Dec 11 03:31 & Dec 10 17:22 & 7930 & $0.13-1.25$ & $115-279$ & 1.94 & -25.8 \\
\hline- & - & - & Dec 11 17:15 & - & $0.58-4.02$ & $122-314$ & 1.30 & - \\
\hline 4 & 1998 & Feb 14 02:29 & Feb 12 17:33 & 8156 & $0.30-0.66$ & $22-30$ & -0.85 & +43.1 \\
\hline 5 & 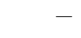 & Apr 29 16:58 & Apr 28 16:38 & 8210 & $0.09-0.83$ & $35-69$ & 1.64 & +26.3 \\
\hline- & - & - & Apr 29 16:39 & - & $0.36-1.19$ & $40-72$ & 1.27 & \\
\hline 6 & 1999 & Feb 14 11:16 & Feb 11 19:26 & 8457 & $0.36-1$ & $43-89$ & -0.46 & -25.0 \\
\hline 7 & 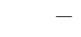 & Aug 04 04:11 & Aug 02 16:36 & 8651 & $0.06-0.13$ & $5-7$ & -1.06 & -37.6 \\
\hline 8 & - & Sep 17 22:28 & Sep 20 17:00 & 8700 & $0-0.53$ & $0-13$ & -0.59 & -124.8 \\
\hline 9 & 2000 & Jul 14 09:27 & Jul 11 16:52 & 9077 & $0.58-0.71$ & $61-69$ & -7.79 & -33.5 \\
\hline- & - & - & Jul 14 16:39 & - & $0.55-0.68$ & $52-112$ & -5.45 & - \\
\hline 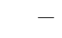 & - & - & Jul 17 16:32 & - & $1.78-6.12$ & $138-274$ & -1.60 & - \\
\hline 10 & - & Jul 25 02:48 & Jul 21 20:28 & 9097 & $0.13-0.14$ & $6.0-6.4$ & -1.42 & -53.9 \\
\hline 11 & - & Jul $2722: 18$ & $\mathrm{Ju}$ & 9097 & $0.19-0.49$ & $40-106$ & 1.42 & -21.6 \\
\hline- & - & - & Jul 28 16:40 & - & $0.83-4.00$ & $121-317$ & -1.14 & 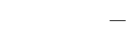 \\
\hline 12 & - & Aug 09 16:30 & Aug 08 16:37 & 9114 & $0.59-1.35$ & $56-79$ & -1.70 & +35.2 \\
\hline- & - & - & Aug 11 16:58 & - & $0.60-1.14$ & $46-62$ & -1.19 & 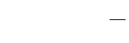 \\
\hline
\end{tabular}

the overlying large-scale dipole alone (the LSD model); and (3) a region of reconnection of the active region and the large-scale dipole (the AR-LSD reconnection model).

First, the large flux ratios are inconsistent with the AR model (If $90 \%$ of the flux from an AR were ejected, there wouldn't be much of an active region left - this is certainly not observed). Large flux ratios are at least consistent with the LSD model and the AR-LSD reconnection model.

That the current ratios tend to be orders of magnitude less than the flux ratios makes sense only in the context of the AR and AR-LSD reconnection models, if there is no significant current present outside the cores of active regions. Although this view is reasonable, it is hard to defend, since as a practical matter vector magnetographs lack sufficient sensitivity to measure currents beyond the strong-field areas of active regions. It remains possible that there exist on the Sun very extended regions outside ARs with current density that is low, but nevertheless not so low that their contribution to the total current of MCs is negligible.

Figure 1 illustrates in graphical form of the proportionality between flux and current. Whether or not the relationship is linear, it is demonstrably real, with Spearman rankorder correlation coefficient of 0.811 at a $99.8 \%$ confidence level. This trend can most easily be explained in terms of the AR-LSD reconnection model. The combination of $I_{M C} / I_{A R}$ and $\Phi_{M C} / \Phi_{A R}$ argues against the AR model.

The quantity $\alpha L$, shown in the last two columns of Table 1 represents the total twist in length $L$. For active regions, we determine $L$ from magnetograms, and for magnetic clouds we take the only directly measured value, $2.5 \mathrm{AU}$ (Larson et al. 1997). It is immediately obvious that $(\alpha L)_{M C} \approx 10(\alpha L)_{A R}$ and that there is no systematic sign or amplitude relationship between $(\alpha L)_{M C}$ and $(\alpha L)_{A R}$. As we have already noted, one-third of the MC-AR pairs have mismatched senses of twist.

$(\alpha L)_{M C} \gg 1$ rules out the AR and LSD models, and only the AR-LSD reconnection model can produce so many turns within the resulting magnetic cloud. Further, only the AR-LSD reconnection model can explain the lack of relationship between $(\alpha L)_{M C}$ and 


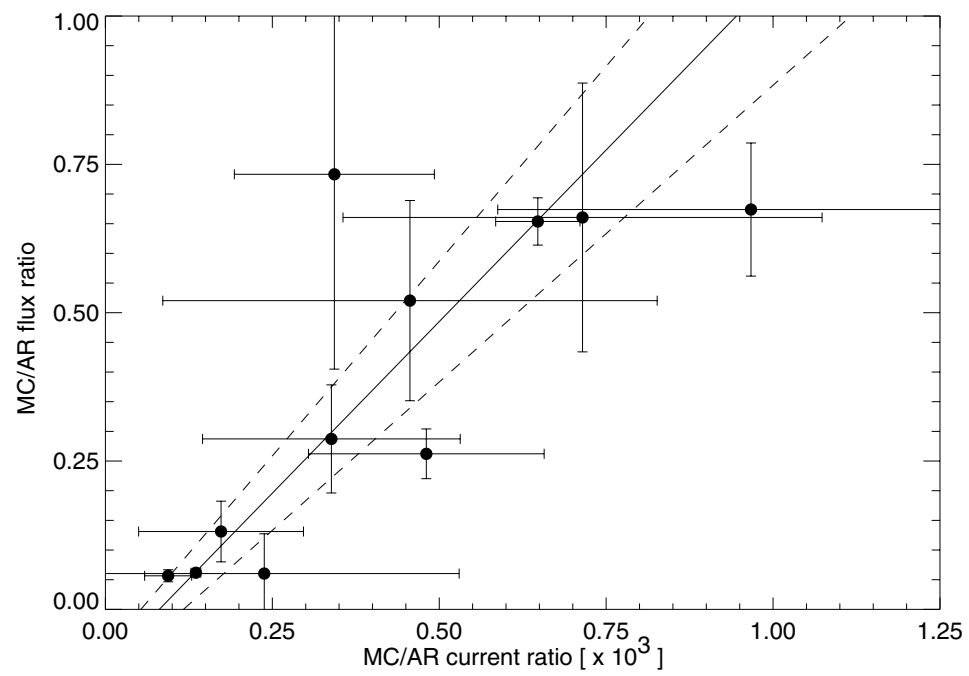

Figure 1. Flux ratio versus current ratio. Reproduced from Leamon et al. (2004, their Figure 3). Whether or not the true relationship is linear, the best fit straight line, with errors, is over-plotted.

$(\alpha L)_{A R}$ (Zhang \& Low 2003). Hence, we are compelled to believe that only models that invoke magnetic reconnection between active regions and their large-scale overlying fields in the eruptive process can explain the relationships between total flux, total current, and associated twist of magnetic fields of magnetic clouds and active regions revealed in this study.

\section{Plasma Structure and Composition}

Charge compositional signatures are very sensitive to identifying ejecta in situ. The best signatures are oxygen and iron charge states (Cane \& Richardson 2003; Lepri \& Zurbuchen 2004):

$$
\mathrm{O}^{7+} / \mathrm{O}^{6+}>1 \quad\left\langle Q_{\mathrm{Fe}}\right\rangle>12 \quad \mathrm{Fe}^{16+} / \mathrm{Fe}_{\text {total }}>0.01
$$

An enhanced alpha/proton ratio is also indicative of ejecta, but at a weaker confidence level and is not unique (for example, it is also indicative of coronal holes).

Higher charge states are indication of a hotter corona at time of freezing-in. They are a better measure of thermal history than the proton temperature observed at 1AU, which has undergone changes due to ejecta evolution, possible in situ heating, and of course depends on the pre-existing solar wind conditions into which the CME was launched. $\mathrm{O}^{7+} / \mathrm{O}^{6+}$ freezes in at $\sim 1 R_{\odot}$, whereas $\left\langle Q_{\mathrm{Fe}}\right\rangle$ is fixed at $3-4 R_{\odot}$ (i.e., at lower temperatures) Enhanced $\mathrm{O}^{7+} / \mathrm{O}^{6+}$ (i.e., hotter) is observed in large flare events and correlates with BDEs (Reinard \& Fisk 2004).

The 'Breakout' model of Antiochos (1998) predicts that if the flux rope forms as result of flare reconnection, one would expect hot ejecta in the center of the cloud. On the other hand, if the flux rope forms before flare onset (e.g., Lin \& Forbes 2000) one would expect a relatively cool flux rope with hot sheaths before and after. The only test of this hypothesis to date (Lynch et al. 2003, their Figure 9) is inconclusive. They observe cooler ends to a relatively uniform $Q_{\mathrm{Fe}}$ profile, but there is no systematic profile in oxygen. 

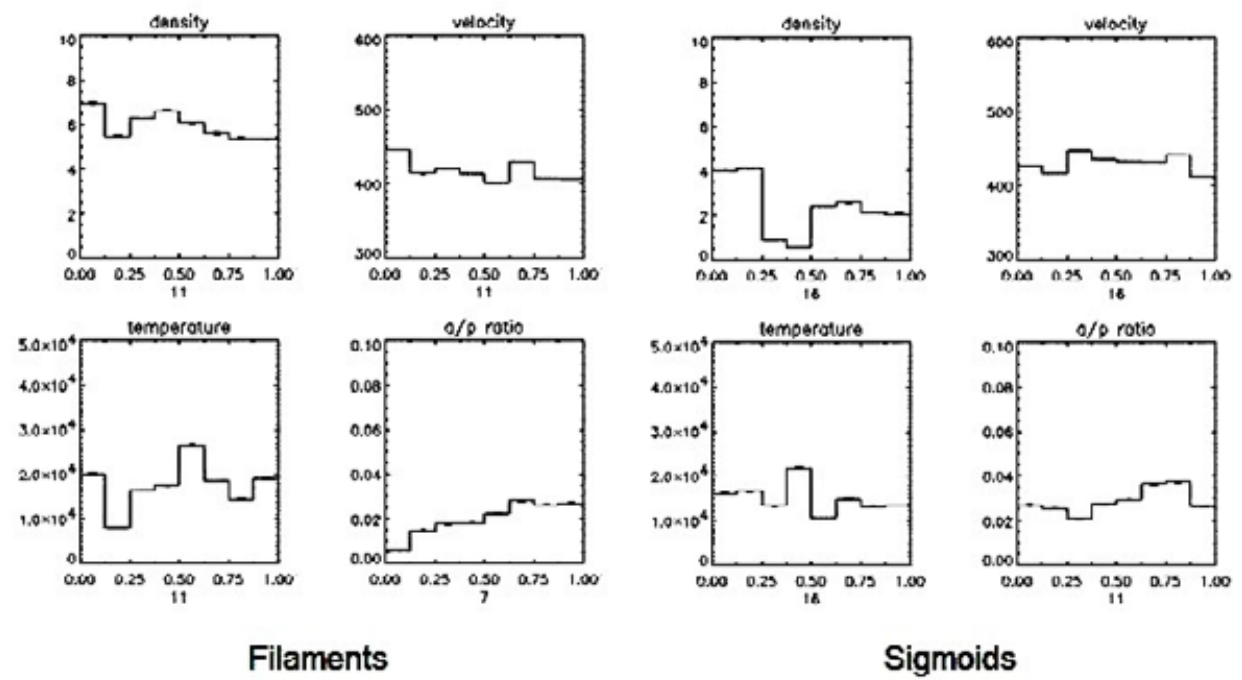

Figure 2. Epoch superposition and weighted averaging of the plasma structure of magnetic clouds associated with active region flares and filament eruptions. There are 11 filament-sourced clouds (of which only 7 have composition data) and 16 AR-sourced clouds (11 with composition data).

Finally, Figure 2 shows tentative first results from a recent study (Leamon, McKenzie and Wilson, "Geoeffective CMEs, Filaments, and Sigmoids", in preparation) suggesting that these tests are indicative of more differences between sigmoids and filaments. Specifically, compare the radically different density and $\alpha / p$ ratio profiles, which suggest different creation mechanisms. (We are in the process of extending these results by including iron and oxygen tests.)

\section{Summary}

Considering 3 simple models - that magnetic cloud flux originates in: (1) active regions alone; (2) the overlying large-scale dipole alone; and (3) a region that has experienced reconnection of the active region and the large-scale dipole, the observational results of the ratios of flux, current and twist only allow the AR-LSD reconnection model to escape unscathed.

Compositional tests, such as those based on iron and oxygen charge states, are sensitive in identifying in situ ejecta. However, there is insufficient statistically significant data to prove or disprove either Antiochos' or Lin \& Forbes' reconnection models.

Filament-related MCs have identifiably different compositional signatures. More study is needed to categorically disprove whether such MCs might be formed either by the LSD model or from pre-existing chromospheric structures.

\section{Acknowledgements}

Funding for this work was provided by AFOSR under grant number F49620-00-1-0128 and the MURI grant to the University of California, Berkeley, and NASA under SR\&T grant NAG5-6110. I would like to express thanks to Dick Canfield, Alexei Pevtsov and Dave McKenzie. More thanks are due to students Keith Lambkin, Brian Lundberg, Sarah Jones and Karen Wilson, all of whom participated in this project through the Research 
Experience for Undergraduates (REU) Program at Montana State University, which is supported by the National Science Foundation under Grant No. 0243923.

Travel assistance to the Beijing IAU symposium from the NSF is also gratefully acknowledged.

\section{References}

Antiochos, S.K. 1998, ApJ 502, L181

Bothmer, V. \& Rust, D.M. 1997, in Geophysical Monograph Series, Vol. 99, Coronal Mass Ejections, ed. N.U. Crooker, J.-A. Joselyn, \& J. Feynman (Washington, D.C.: American Geophysical Union), 139

Burlaga, L.F., Sittler, E.C., Mariani, F., \& Schwenn, R. 1981, J. Geophys. Res., 86, 6673

Cane, H.V. \& Richardson, I.G. 2003, J. Geophys. Res. 108, 6

Crooker, N.U. 2000, J. Atmos. and Solar-Terr. Phys. 62, 1071

Gosling, J.T. 1990, in Geophysical Monograph Series, Vol. 58, Physics of Magnetic Flux Ropes, ed. C.T. Russell, E.R. Priest, \& L.C. Lee (Washington, D.C.: American Geophysical Union), 343

Larson, D.E., Lin, R.P., McTiernan, J.M., McFadden, J.P., Ergun, R.E., McCarthy, M., Rème, H., Sanderson, T.R., Kaiser, M., Lepping, R.P., \& Mazur, J. 1997, Geophys. Res. Lett. 24, 1911

Leamon, R.J., Canfield, R.C., Jones, S.L., Lambkin, K., Lundberg, B.J., \& Pevtsov, A.A. 2004, J. Geophys. Res. 109, 5106, doi:10.1029/2003JA010324

Leamon, R.J., Canfield, R.C., \& Pevtsov, A.A. 2002, J. Geophys. Res. 107, 1234, doi:10.1029/2001JA000313

Lepping, R.P., Jones, J.A., \& Burlaga, L.F. 1990, J. Geophys. Res. 95, 11957

Lepri, S.T. \& Zurbuchen, T.H. 2004, J. Geophys. Res. 109, 1112

Lin, J. \& Forbes, T.G. 2000, J. Geophys. Res. 105, 2375

Lundquist, S. 1950, Ark. Fys. 2, 361

Lynch, B.J., Zurbuchen, T.H., Fisk, L.A., \& Antiochos, S.K. 2003, J. Geophys. Res. 108, 1239, doi:10.1029/2002JA009591

Pevtsov, A.A. \& Canfield, R.C. 2001, J. Geophys. Res. 106, 25191

Pevtsov, A.A., Canfield, R.C., \& Metcalf, T.R. 1995, ApJ 440, L109

Reinard, A.A. \& Fisk, L.A. 2004, ApJ 608, 533

Webb, D.F. 2000, IEEE Trans. Plasma Sci. 28, 1795

Zhang, M. \& Low, B.C. 2003, ApJ 584, 479

\section{Discussion}

JINGXIU WANG: Your results are consistent with the observations at the solar surface. Although many CMEs are initiated from active regions, a favorite large-scale magnetic structure is important for the occurrence of a CME. But can you say something about the characteristics of your large-scale dipoles, have you made some studies on these largescale structures?

LEAMON: In this work, we only argue the need for reconnection with the large-scale fields. We have made some tentative studies comparing the mutual helicity of an active region and the overlying fields from, say, a PFSS ('hairy ball') model, but they are only preliminary and not conclusive.

STERLING: Several folks, including myself, have so far used only a qualitative morphological definition of sigmoids. At some point, we should improve upon this. What definition did you use for sigmoids? 
LEAMON: I, too, only used a qualitative, morphological definition. Perhaps it would have been better to say "active region events" or "flare-related events" and to differentiate them from "quiescent filament events."

P.F. CHEn: Generally CME modeling involves magnetic reconnection below the erupting flux-rope, which leads to small-scale solar flares. Could you explain magnetic reconnection between active region and large-scale surroundings in detail?

LEAMON: Like my answer to Jingxiu Wang, the conclusions of this work are that the flux-rope magnetic cloud observed at $1 \mathrm{AU}$ must be formed in the course of eruption, by reconnection between the active region and overlying fields. Clearly the next step is to model that reconnection quantitatively, but we have not done that yet. 